Proceedings of the

International Geometry Center

Vol. 11, no. 2 (2018) pp. 1-15

\title{
A calculation of periodic data of surface diffeomorphisms with one saddle orbit
}

\author{
Elena Nozdrinova, Olga Pochinka
}

\begin{abstract}
We prove that every orientable surface admits an orientationpreserving diffeomorphism with one saddle orbit. It distinguishes in principle the considered class of systems from source-sink diffeomorphisms existing only on the sphere. It is shown that diffeomorphisms with one saddle orbit of a positive type on any surface have exactly three node orbits. We also describe all possible types of periodic data for such diffeomorphisms, and found formulas expressing periods of sources via periods of the sink and the saddle.
\end{abstract}

Анотація. При вивченні дискретних динамічних систем важливу роль відіграють періодичні орбіти. Класичним прикладом є теорема Шарковського про відображення відрізку в себе, яка стверджує, що з існування орбіт періоду три "породжуе хаос". В останні 40 років з'явилось багато робіт присвячених вивченню періодичних даних відображень поверхонь. Найбільш корисними інструментами для доведення існування нерухомих точок та, в більш загальному випадку, періодичних точок неперервного відображення компактного многовиду, є теорема Лефшеця про нерухому точку та їі узагальнення. Дзета-функція Лефшеця спрощує вивчення періодичних точок дифеоморфізмів поверхонь з регулярною динамікою. Результати досліджень в даному напрямку можна знайти в роботах таких авторів як: П. Бланшар, С. Баттерсон, У. Жако, Дж. Френкс, С. Нарасімхан і ін. Опис періодичних даних градієнтноподібних дифеоморфізмів поверхонь був отриманий А. Безденежних та В. Грінесом і спирався на класифікацію гомеоморфізмів поверхонь, отриману Дж. Нільсеном.

У роботі "A complete topological classification of Morse-Smale diffeomorphisms on surfaces: a kind of kneading theory in dimension two" В. Грінес, О. Починка, С. Ван Стрієн показали, що вивчення періодичних даних довільних дифеоморфізмів Морса-Смейла на поверхнях зводиться шляхом фільтрації до задачі обчислення періодичних даних дифеоморфізмів з єдиною седловою періодичною орбітою. Представлена робота присвячена вирішенню останньої задачі у випадку, коли орбіта сідлової точки

Keywords: periodic data, surface 
має додатний тип орієнтації. В статті доведено, що на кожній орієнтовній поверхні існує дифеоморфізм, який зберігає орієнтацію і має єдину сідлову орбіту. Це принципово відрізняє розглянутий клас систем від дифеоморфізмів "джерело-стік", які існують лише на сфері. Показано, що дифеоморфізми з однією сідловою орбітою на будь-якій поверхні мають рівно три вузлових орбіти. Крім того, встановлено всі можливі типи періодичних даних для таких дифеоморфізмів, а саме: знайдено формули, що явно виражають періоди джерел через періоди стоку та сідла.

\section{INTRODUCTION AND A FORMULATION OF RESULTS}

In the study of discrete dynamical systems, i.e. study of orbits of selfmaps $f$ defined on a given compact manifold, the periodic behavior plays an important role. During the last forty years there were a growing number of results showing that certain simple assumptions on $f$ force qualitative and quantitative properties (like the set of periods) of a system. One of the best known result in this direction is the paper entitled "Period three implies chaos for the interval continuous self-maps" (see, for example, [8]). The effect described in [8] was discovered by A. Sharkovsky in [12]. The most useful tools for proving existence of fixed points, or more generally of periodic points for a continuous self-map $f$ of a compact manifold, is the Lefschetz Fixed Point Theorem and its generalizations (see, for instance [11], [3]). The Lefschetz zeta function simplifies the study of periodic points of $f$. This is a generating function for all the Lefschetz numbers of all iterates of $f$.

Periodic data of diffeomorphisms with regular dynamics on surfaces is studied in already classical works by P. Blanchard, J. Franks, R. Bowen, S. Batterson, J. Smillie, W. Jaco, P. Shalen, C. Narasimhan and other. Description of periodic data of gradient-like diffeomorphisms of surfaces was given by A. Bezdenezhnykh and V. Grines [1], using J. Nielsen's classification of periodic surface transformations. In the paper by V. Grines, O. Pochinka, S. Van Strien [6] it was shown that the study of periodic data of arbitrary Morse-Smale diffeomorphisms on surfaces is reduced by filtration to the problem of computing periodic data of diffeomorphisms with a unique saddle periodic orbit. The present paper is devoted to a solution of this problem.

As first we will recall some basic definitions and notations.

Let $S_{g}$ be a closed orientable surface of genus $g \geq 0$ with a metric $d$ and $f: S_{g} \rightarrow S_{g}$ be an orientation preserving diffeomorphism. A point $x \in S_{g}$ is called wandering for $f$, if there exists an open neighborhood $U_{x}$ of $x$ such that $f^{n}\left(U_{x}\right) \cap U_{x}=\emptyset$ for all $n \in \mathbb{N}$. Otherwise, $x$ is non-wandering. 
The set of all non-wandering points of $f$ is denoted by $\Omega_{f}$ and called the non-wandering set of $f$.

When $\Omega_{f}$ is finite then every point $p \in \Omega_{f}$ is periodic, and its period will be denoted by $m_{p} \in \mathbb{N}$. Moreover, $p$ is called hyperbolic if the Jacobian matrix $\left.\left(\frac{\partial f^{m} p}{\partial x}\right)\right|_{p}$ has eigenvalues whose absolute values are distinct from 1 . If the absolute values of all eigenvalues are less (resp. greater) than 1, then $p$ is called a sink (resp. source). Sink and source points are also called nodes, while hyperbolic periodic points being not nodes are called saddles.

Hyperbolicity of a periodic point $p$ leads to the existence of the stable, $W_{p}^{s}$, and unstable, $W_{p}^{u}$, manifolds, which are defined as follows:

$$
\begin{aligned}
& W_{p}^{s}=\left\{x \in S_{g}: \lim _{k \rightarrow+\infty} d\left(f^{k \cdot \operatorname{per}(p)}(x), p\right)=0\right\}, \\
& W_{p}^{u}=\left\{x \in S_{g}: \lim _{k \rightarrow+\infty} d\left(f^{-k \cdot \operatorname{per}(p)}(x), p\right)=0\right\} .
\end{aligned}
$$

The stable and unstable manifolds are called invariant manifolds. A connected component of the set $W_{p}^{u} \backslash p\left(\right.$ resp. $\left.W_{p}^{s} \backslash p\right)$ is called unstable (resp. stable) separatrix.

A diffeomorphism $f: S_{g} \rightarrow S_{g}$ is Morse-Smale if the set of its wandering points consists of finitely many periodic points and there is no separatrixes connecting saddles.

To the orbit $\mathcal{O}_{p}$ of a periodic point $p$ of a Morse-Smale diffeomorphism $f$ one can associate the following numbers $\left(m_{p}, q_{p}, \nu_{p}\right)$ called the periodic data of $p$, where

- $m_{p}$ is the period of $p$,

- $q_{p}=\operatorname{dim} W_{p}^{u}$, and

- $\nu_{p}$ is the orientation type of $p$ which equals +1 (resp. -1) whenever $\left.f^{m_{p}}\right|_{W_{p}^{u}}$ preserves (resp. reverses) orientation.

Denote by $G\left(S_{g}\right)$ the set of Morse-Smale diffeomorphisms $f: S_{g} \rightarrow S_{g}$ having a unique saddle periodic orbit $\mathcal{O}_{\sigma}$ and satisfying $\nu_{\sigma}=+1$. The case $\nu_{\sigma}=-1$ was investigated in [9].

Let $f \in G\left(S_{g}\right)$. It is well known that the Euler characteristic for an orientable surface of genus $g$ is expressed by the formula: $\chi\left(S_{g}\right)=2-2 g$, (see, for example, [5], [2]). On the other hand, by [13], a Morse-Smale diffeomorphism induces a cellular decomposition of $S_{g}$ whose open cells are unstable submanifolds of periodic points:

$$
S_{g}=\bigcup_{p \in \Omega_{f}} W_{p}^{u} .
$$

Then

$$
c_{2}-c_{1}+c_{0}=2-2 g
$$


where $c_{2}$ is be the number of the sources of $f$ corresponding to 2-cells, $c_{1}$ is the number saddles corresponding to 1-cells, and $c_{0}$ is the number of sinks (0-cells).

The following theorem describes the numbers of the periodic orbits of $f \in G\left(S_{g}\right)$.

Theorem 1.1. The non-wandering set of every diffeomorphism $f \in G\left(S_{g}\right)$ consists of a unique saddle orbit and three node orbits: either one sink orbit and two source orbits or one source orbit and two sink orbits.

In what follows we assume that a diffeomorphism $f \in G\left(S_{g}\right)$ has a unique sink orbit $\mathcal{O}_{\omega}$ and two source orbits $\mathcal{O}_{\alpha_{1}}, \mathcal{O}_{\alpha_{2}}$ (otherwise, we could just replace $f$ with its inverse $f^{-1}$ ).

By assumption $f$ has one saddle periodic orbit preserving the orientation, hence $\nu_{\sigma}=+1$ and $q_{\sigma}=1$. The orbits of $\mathcal{O}_{\alpha_{1}}, \mathcal{O}_{\alpha_{2}}$ consist of sources, so $q_{\alpha_{1}}=q_{\alpha_{2}}=\operatorname{dim} W_{\alpha_{i}}^{u}=2$. Moreover, since $f$ preserves the orientation of open connected invariant subsets, we get that $\nu_{\alpha_{i}}=+1$. The orbit of $\mathcal{O}_{\omega}$ consists of sinks, whence $q_{\omega}=\operatorname{dim} W_{\omega}^{u}=0$ and $\nu_{\omega}=+1$. Thus, a part of the periodic data is already known and our task is to find the periods $m_{\omega}, m_{\sigma}, m_{\alpha_{1}}, m_{\alpha_{2}}$. Notice that from (1.1) we have

$$
m_{\alpha_{1}}+m_{\alpha_{2}}-m_{\sigma}+m_{\omega}=2-2 g .
$$

Below $(a, b)$ means the greatest common divisor of the natural numbers $a, b$, also we assume $(0, b)=b$.

Theorem 1.2. Every diffeomorphism $f \in G\left(S_{g}\right)$ has the following periodic data:

$$
\begin{aligned}
m_{\omega} & =m, \quad m_{\sigma}=k m, \\
m_{\alpha_{1}} & =(k, j+1)\left(\frac{k}{(k, j+1)}, m\right), \\
m_{\alpha_{2}} & =(k, j)\left(\frac{k}{(k, j)}, m\right),
\end{aligned}
$$

for some $m \in \mathbb{N}, k \in \mathbb{N}, j \in\{0, \ldots, k-1\}$.

Furthermore, for every collection of the natural numbers $k \in \mathbb{N}, m \in \mathbb{N}$, $j \in\{0, \ldots, k-1\}$ there exists a diffeomorphism $f \in G\left(S_{g}\right)$ with a periodic data of the form (1.3) on a surface of genus

$$
g=1+\frac{1}{2}\left((k-1) m-(k, j+1)\left(\frac{k}{(k, j+1)}, m\right)-(k, j)\left(\frac{k}{(k, j)}, m\right)\right) .
$$

Corollary 1.3. Due to theorem above every orientable surface of genus $g$ admits a diffeomorphism from the class $G\left(S_{g}\right)$, with the following periodic data: $m_{\sigma}=2 g+1, m_{\omega}=m_{\alpha_{1}}=m_{\alpha_{2}}=1$, see Figure 1.1. 


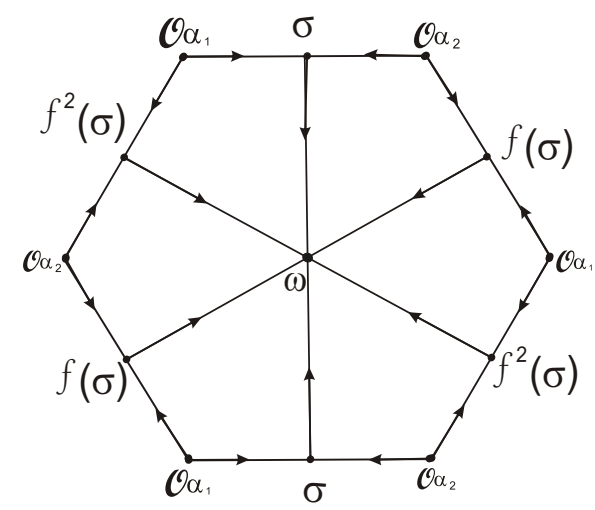

FIGURE 1.1. Diffeomorphisms from $G\left(S_{1}\right)$ with $m_{\sigma}=3$, $m_{\omega}=m_{\alpha_{1}}=m_{\alpha_{2}}=1$

Acknowledgements. The study of the non-wandering set structure of considered diffeomorphisms was financial supported by RFBR (project 1831-00022), the finding of a list of the periodic dates for these diffeomorphisms was supported by the fundamental research program of the HSE in 2018.

The authors are grateful to the referee for the careful reading of the manuscript and very useful comments.

\section{Structure of The NON-WANDERING SET}

In this section we prove Theorem 1.1. Detailed proofs of auxiliary statements given in this section can be found in [5].

Proof. Let us show that the non-wandering set of every diffeomorphism $f \in G\left(S_{g}\right)$ consists of a unique saddle orbit and three node orbits (either one sink and two source, or one source and two sink orbits).

For the saddle separatrix $\ell$ we denote by $m_{\ell}$ its period, that is, the smallest natural number $\mu$ such that $f^{\mu}(\ell)=\ell$. Also denote by $\mathcal{O}_{\ell}$ the orbit of the separatrix $\ell$. By [5, Proposition 2.3, p. 31], the closure of each unstable saddle separatrix contains a unique sink, while the closure of each stable saddle separatrix contains a unique source. Assume that the unstable separatrix $\ell^{u}$ of a saddle point $\sigma$ contains a sink $\omega$ in its closure. Let $m$ be the period of $\omega$. According to [10, Theorem 5.5], $f^{m}$ is locally conjugate at the point $\omega$ with the linear diffeomorphism of $\mathbb{R}^{2}$ given by the formula

$$
L(x, y)=\left(\frac{x}{2}, \frac{y}{2}\right)
$$


Let $\mathcal{O}_{\omega}$ be the orbit of the point $\omega, V_{\omega}=W_{\mathcal{O}_{\omega}}^{s} \backslash \mathcal{O}_{\omega}, \hat{V}_{\omega}=V_{\omega} / f$ be the orbit space of the action of the group $F=\left\{f^{k}, k \in \mathbb{Z}\right\}$ on $V_{\omega}$, and $p_{\omega}: V_{\omega} \rightarrow \hat{V}_{\omega}$ the natural projection.

Notice that $V_{\omega}$ is diffeomorphic to a disjoint union of $m$ open cylinders $S^{1} \times \mathbb{R}$ that are cyclically interchanged by $f$. Moreover, due to [5, Proposition 2.5, p. 35], the space $\hat{V}_{\omega}$ is diffeomorphic to a two-dimensional torus. Let $x \in V_{\omega}$ be any point and $\hat{x}=p_{\omega}(x)$. Then the natural projection $p_{\omega}: V_{\omega} \rightarrow \hat{V}_{\omega}$ is a covering map and we have the following exact sequence

$$
0 \rightarrow \pi_{1}\left(V_{\omega}, x\right) \rightarrow \pi_{1}\left(\hat{V}_{\omega}, \hat{x}\right) \stackrel{\eta_{\omega}}{\longrightarrow} \pi_{0} \mathbb{Z} \rightarrow \pi_{0} V_{\omega} \rightarrow 1,
$$

where $\eta_{\omega}$ is the boundary homomorphism. Since the connected component of $x$ in $V_{\omega}$ consists of points $\left\{f^{k m_{\omega}}(x)\right\}_{k \in \mathbb{Z}}$, it follows that the image of $\eta_{\omega}$ is a subgroup $m_{\omega} \mathbb{Z} \subset \mathbb{Z} \cong \pi_{0} \mathbb{Z}$.

In other words, we get an epimorphism $\eta_{\omega}: \pi_{1}\left(\hat{V}_{\omega}\right) \rightarrow m_{\omega} \mathbb{Z}$ onto the subgroup of $\mathbb{Z}$ consisting of multiples of $m_{\omega}$. For the convenience of the reader let us recall the definition of $\eta_{\omega}$. Let $[\hat{c}] \in \pi_{1}\left(\hat{V}_{\omega}, \hat{x}\right)$ be a loop in $\hat{V}_{\omega}$ and $c:[0,1] \rightarrow V_{\omega}$ be its lift starting at $c(0)=x \in V_{\omega}$. Then the end point $c(1)=f^{n}(x)$, for some $n \in m_{\omega} \mathbb{Z}$, and $\eta_{\omega}([\hat{c}])=n$.

Denote by $a: \mathbb{R}^{2} \rightarrow \mathbb{R}^{2}$ the diffeomorphism given by the formula

$$
a(x, y)=\left(\frac{x}{2}, 2 y\right) .
$$

Evidently, it has a unique fixed saddle point at the origin $O$ with the stable manifold $W_{O}^{s}=O x$ and the unstable manifold $W_{O}^{u}=O y$. Then the diffeomorphism $f^{m_{\sigma}}$ in some neighborhood of the point $\sigma$ is topologically connected to the diffeomorphism $a$ in a neighborhood of the point $O$ (see, for example, [10, Theorem 5.5]).

Let $\hat{\ell}^{u}=p_{\omega}\left(\ell^{u}\right)$ and $j_{\hat{\ell}^{u}}: \hat{\ell}^{u} \rightarrow \hat{V}_{\omega}$ be the inclusion map. It follows from [5, Proposition 2.5, p.35] that the set $\hat{\ell}^{u}$ is a circle smoothly embedded in $\hat{V}_{\omega}$ and such that $\eta_{\omega}\left(j_{\hat{\ell}^{u}}\left(\pi_{1}\left(\hat{\ell}^{u}\right)\right)\right)=m_{\ell^{u}} \mathbb{Z}$. Notice that $p_{\omega}\left(\mathcal{O}_{\ell^{u}}\right)=\hat{\ell}^{u}$.

Figure 2.1 depicts the torus $\hat{V}_{\omega}$ with the projection $\hat{\ell}^{u}$ of the separatrix $\ell^{u}$ such that $\frac{m_{\ell} u}{m_{\omega}}=3$.

Let $\mathcal{N}=\left\{(x, y) \in \mathbb{R}^{2}:|x y| \leq 1\right\}$. Notice that the set $\mathcal{N}$ is invariant with respect to the diffeomorphism $a$. A neighborhood of $N_{\sigma}$ of the point $\sigma$ is called linearizing if there exists a homeomorphism $\mu_{\sigma}: N_{\sigma} \rightarrow \mathcal{N}$ conjugating the restriction $\left.f^{m_{\sigma}}\right|_{N_{\sigma}}$ with the diffeomorphism $\left.a\right|_{\mathcal{N}}$.

In this case the neighborhood

$$
N_{\mathcal{O}_{\sigma}}=\bigcup_{j=0}^{m_{\sigma}-1} f^{j}\left(N_{\sigma}\right)
$$




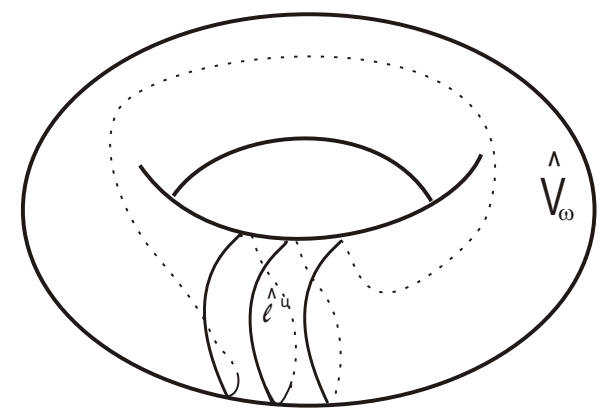

FiguRE 2.1. The projection of the saddle separatrix in the orbit space of the sink basin homeomorphic to the torus

of the orbit $\mathcal{O}_{\sigma}=\bigcup_{j=0}^{m_{\sigma}-1} f^{j}(\sigma)$ equipped with the map $\mu_{\mathcal{O}_{\sigma}}: N_{\mathcal{O}_{\sigma}} \rightarrow \mathcal{N}$, defined by

$$
\left.\mu_{\mathcal{O}_{\sigma}}\right|_{f^{j}\left(N_{\sigma}\right)}=\mu_{\sigma} \circ f^{-j}: f^{j}\left(N_{\sigma}\right) \rightarrow \mathcal{N}, \quad j=0, \ldots, m_{\sigma}-1,
$$

is called the linearizing neighborhood of the orbit $\mathcal{O}_{\sigma}$.

Due to [5, Theorem 2.2, p. 29], the saddle point (orbit) of the diffeomorphism $f$ has a linearizing neighborhood.

Let $\mathcal{N}^{u}=\mathcal{N} \backslash O x$ and $\hat{\mathcal{N}}^{u}=\mathcal{N}^{u} / a$ be the orbit space of the action of the group $\left\{a^{n}, n \in \mathbb{Z}\right\}$ on $\mathcal{N}^{u}$. Then the natural projection $p_{\hat{\mathcal{N}} u}: \mathcal{N}^{u} \rightarrow \hat{\mathcal{N}}^{u}$ is a covering map. Moreover, the fundamental domain of the action of the group $\left\{a^{n}, n \in \mathbb{Z}\right\}$ on $\mathcal{N}^{u}$ consists of two disjoint curvilinear trapezoids, each of which has equivalent points belonging to the horizontal segments of the boundary. In Figure 2.2 these trapezoids are shaded and it is shown how we can obtain the manifold $\hat{\mathcal{N}}^{u}$ by identifying their boundaries via the diffeomorphism $a$. Thus the space $\hat{\mathcal{N}}^{u}$ is homeomorphic to a pair of two-dimensional annuli $K_{1}, K_{2}$.

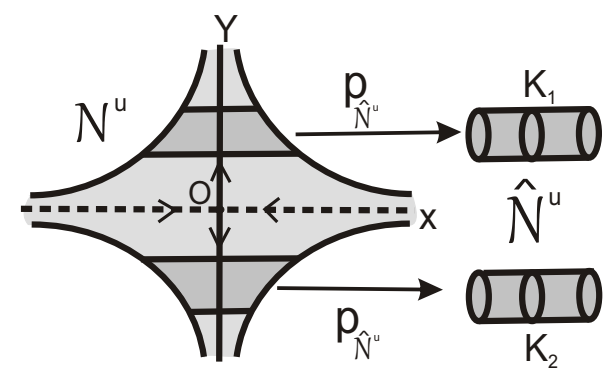

FIGURE 2.2. The orbit space $\hat{\mathcal{N}}^{u}$ 
Let

$$
N_{\sigma}^{u}=N_{\sigma} \backslash W_{\sigma}^{s}, \quad N_{\sigma}^{s}=N_{\sigma} \backslash W_{\sigma}^{u} .
$$

Denote by $N_{\ell^{u}}$ the connected component of the set $N_{\sigma}^{u}$ containing an unstable separatrix $\ell^{u}$. Let also $\hat{N}_{\ell^{u}}=p_{\omega}\left(N_{\ell^{u}}\right)$ and $j_{\hat{\ell}^{u}}: N_{\hat{\ell}^{u}} \rightarrow \hat{V}_{\omega}$ be the inclusion map. The set $\hat{N}_{\ell^{u}}$ is a smoothly embedded annulus in $\hat{V}_{\omega}$ such that $\eta_{\omega}\left(j_{\hat{N}_{\ell^{u}} *}\left(\pi_{1}\left(\hat{N}_{\ell^{u}}\right)\right)\right)=m_{\ell^{u}} \mathbb{Z}$.

Denote by $A$ the union of all sink points of the diffeomorphism $f$. Let also $V_{A}=W_{A}^{s} \backslash A, \hat{V}_{A}=V_{A} / f$, and $p_{A}: V_{A} \rightarrow \hat{V}_{A}$ be the natural projection. Similarly to the above arguments, the orbit space in the sink basin is homeomorphic to the torus, which implies that each connected component of the set $\hat{V}_{A}$ is homeomorphic to a two-dimensional torus, and the number of connected components coincides with the number of sink orbits.

Let $N_{\mathcal{O}_{\sigma}}^{u}=N_{\mathcal{O}_{\sigma}} \backslash W_{\mathcal{O}_{\sigma}}^{s}, N_{\mathcal{O}_{\sigma}}^{s}=N_{\mathcal{O}_{\sigma}} \backslash W_{\mathcal{O}_{\sigma}}^{u}, \hat{N}_{\mathcal{O}_{\sigma}}^{u}=N_{\mathcal{O}_{\sigma}}^{u} / f$. It follows from [5, Theorem 2.4, p. 42] that the set $\hat{N}_{\mathcal{O}_{\sigma}}^{u}$ is a pair of annuli smoothly embedded in $\hat{V}_{A}$. Also due to [5, Corollary 2.1, p. 46] the set $\hat{V}_{A}$ is not empty and, by [5, Corollary 2.2 , p. 62 ], each torus in $\hat{V}_{A}$ has to contain at least one annulus from the set $\hat{N}_{\mathcal{O}_{\sigma}}^{u}$. Thus $\hat{V}_{A}$ contains one or two connected components.

Similar statements can be formulated for the source point $\alpha$ and for the stable separatrix $\ell^{s}$ of the saddle point $\sigma$ such that $\ell^{s} \subset W_{\alpha}^{u}$.

Denote by $R$ the union of the source points of the diffeomorphism $f$. Let also $V_{R}=W_{R}^{u} \backslash R, \hat{V}_{R}=V_{R} / f$ and $p_{R}: V_{R} \rightarrow \hat{V}_{R}$ be the natural projection. Similarly to the above arguments, the orbit space in the source basin is homeomorphic to the torus, which implies that each connected component of the set $\hat{V}_{R}$ is homeomorphic to a two-dimensional torus, and the number of connected components coincides with the number of source orbits. On the other hand, it follows from the equality $V_{R}=\left(V_{A} \backslash N_{\mathcal{O}_{\sigma}}^{u}\right) \cup N_{\mathcal{O}_{\sigma}}^{s}$ (see, for instance, [5, Theorem 2.1, p. 28]) that

$$
\hat{V}_{A}=\left(\hat{V}_{R} \backslash \hat{N}_{\mathcal{O}_{\sigma}}^{u}\right) \cup \hat{N}_{\mathcal{O}_{\sigma}}^{s} .
$$

Thus, to get the space $\hat{V}_{R}$ we have to delete $\hat{N}_{\mathcal{O}_{\sigma}}^{u}$ from the torus $\hat{V}_{A}$ and glue the set $\hat{N}_{\mathcal{O}_{\sigma}}^{s}$ to the boundary of the resulting set.

Each of the sets $\hat{N}_{\mathcal{O}_{\sigma}}^{u}, \hat{N}_{\mathcal{O}_{\sigma}}^{s}$ consists of two annuli. Moreover, the annuli $\hat{N}_{\mathcal{O}_{\sigma}}^{u}$ are homotopically non-trivially embedded in the torus $\hat{V}_{A}$. If we assume that $\hat{V}_{A}$ consists of a unique connected component then $\hat{V}_{A} \backslash \hat{N}_{\mathcal{O}_{\sigma}}^{u}$ consists of two annuli and a gluing $\hat{N}_{\mathcal{O}_{\sigma}}^{s}$ to their boundaries gives two twodimensional tori (see Figure 2.3, where the transition from the sink basins to the sources basins is illustrated by the example of a diffeomorphism 

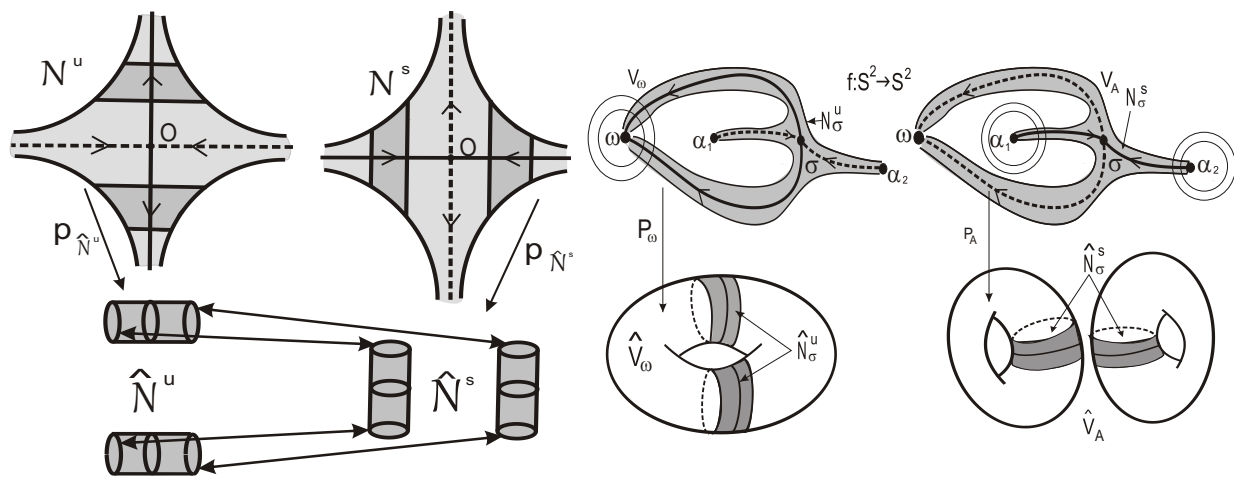

FiguRE 2.3. Regluing along annuli

of the 2-sphere. For convenience, in the above-mentioned basins, fundamental regions are selected, after the identifying of their boundary circles the corresponding tori in the quotient spaces are obtained). This means that there are exactly two source orbits for the diffeomorphism $f$, that is, $R=\mathcal{O}_{\alpha_{1}} \cup \mathcal{O}_{\alpha_{2}}$ for some periodic sources $\alpha_{1}, \alpha_{2}$.

If we assume that $\hat{V}_{A}$ consists of two connected components then the similar cut and gluing operation implies the existence of the unique source orbit in this case.

\section{Periodic Data}

This section is devoted to the proof of Theorem 1.2. Firstly let us show that every diffeomorphism $f \in G\left(S_{g}\right)$ has following periodic data, see (1.3):

$$
\begin{aligned}
& m_{\omega}=m, \quad m_{\sigma}=k m, \\
& m_{\alpha_{1}}=(k, j+1)\left(\frac{k}{(k, j+1)}, m\right), \\
& m_{\alpha_{2}}=(k, j)\left(\frac{k}{(k, j)}, m\right),
\end{aligned}
$$

where $m \in \mathbb{N}, k \in \mathbb{N}, j \in\{0, \ldots, k-1\}$ are natural numbers.

Let us introduce an abstract model of dynamics in the basin of a periodic sink of period $m$. Let $m \geq 1$ be an integer and $V_{m}=\mathbb{S}^{1} \times \mathbb{R}^{+} \times \mathbb{Z}_{m}$. Thus $V_{m}$ is a model for the basin of a periodic sink of period $m$. Let $k \in \mathbb{N}$, $\tau \in\{0, \ldots, k-1\}$ and

$$
\gamma_{1}^{\tau}=\bigcup_{\tau=0}^{k-1} e^{i \pi\left(\frac{1}{2}-\frac{2 \tau}{k}\right)} \times \mathbb{R}^{+}, \quad \gamma_{2}^{\tau}=\bigcup_{\tau=0}^{k-1} e^{i \pi\left(\frac{1}{2}-\frac{2 \tau+1}{k}\right)} \times \mathbb{R}^{+},
$$




$$
\gamma_{1}=\bigcup_{\tau=0}^{k-1} \gamma_{1}^{\tau} \times \mathbb{Z}_{m}, \quad \gamma_{2}=\bigcup_{\tau=0}^{k-1} \gamma_{2}^{\tau} \times \mathbb{Z}_{m},
$$

Here $\gamma_{1} \cup \gamma_{2}$ models the saddle unstable separatrices, see Figure 3.1.

Let $n \geq 0$ be any integer satisfying the following conditions:

- if $k=1$, then $n=0$;

- otherwise, $n \in\{1, \ldots, k-1\}$ is such that $m n$ and $k$ are co-prime.

Here $m n$ models the period of periodic unstable separatrices in $V_{m}$ and $\frac{m n}{k}$ represents their "rotation number", i.e. how the diffeomorphism permutes these separatrices. As a local model for the diffeomorphism on the basin we take the contraction $\phi_{m, k, n}: V_{m} \rightarrow V_{m}$ given by the formula:

$$
\phi_{m, k, n}(z, r, w)=\left(z e^{-\frac{2 \pi m n}{k} i}, \frac{r}{2^{m}}, w+1 \bmod m\right) .
$$

Then

$$
\phi_{m, k, n}\left(\gamma_{i}^{\tau} \times\{w\}\right)=\gamma_{i}^{\tau+m n} \times\{w+1 \bmod m\}
$$

for all $i=1,2, \tau=0, \ldots, k-1, w=0, \ldots, m-1$.

Notice that $\hat{V}_{m, k, n}=V_{m} / \phi_{m, k, n}$ is a torus. Let $p_{m, k, n}: V_{m} \rightarrow \hat{V}_{m, k, n}$ the natural projection. Then $\hat{\gamma}_{i}=p_{m, k, n}\left(\gamma_{i}\right), i=1,2$ is a knot in $\hat{V}_{m, k, n}$.

Let $f \in G\left(S_{g}\right)$. For the sink orbit $\mathcal{O}_{\omega}$ put $V_{\omega}=W_{\mathcal{O}_{\omega}}^{s} \backslash \mathcal{O}_{\omega}$. Denote by $\hat{V}_{\omega}=V_{\omega} / f$ the orbit space of the action of the group $F=\left\{f^{i}, i \in \mathbb{Z}\right\}$ on $V_{\omega}$ and by $p_{\omega}: V_{\omega} \rightarrow \hat{V}_{\omega}$ the natural projection. The unstable separatrices $\ell_{1}^{u}$, $\ell_{2}^{u}$ of the saddle point $\sigma$ have period $m_{\sigma}$ and lie in the basin $V_{\omega}$. Since the group $F$ acts transitively on the connected components of $V_{\omega}$ (the number of such components is $m$ ) and on the orbit of each unstable separatrix (the number of the connected components of this orbit is $m_{\sigma}$ ), it follows that in each connected component of the set $V_{\omega}$ there exists the same number of separatrices from that orbit. Hence the period $m_{\sigma}$ is a multiple of the period $m$.

Thus each connected component of $V_{\omega}$ contains $k:=\frac{m_{\sigma}}{m}$ separatrices from the orbit of the separatrix $\ell_{i}^{u}$. Let $\hat{\ell}_{1}^{u}=p_{\omega}\left(\ell_{1}^{u}\right)$ and $\hat{\ell}_{2}^{u}=p_{\omega}\left(\ell_{2}^{u}\right)$. Then there is a number $n$ and a diffeomorphism $\hat{h}_{\omega}: \hat{V}_{\omega} \rightarrow \hat{V}_{m, k, n}$ transforming the knots $\hat{\ell}_{1}^{u}, \hat{\ell}_{2}^{u}$ to the knots $\hat{\gamma}_{1}, \hat{\gamma}_{2}$. Thus, there is a lift $h_{\omega}: V_{\omega} \rightarrow V_{m}$ of $\hat{h}_{\omega}$ which sends the separatrices $W_{\mathcal{O}_{\sigma}}^{u} \backslash \mathcal{O}_{\sigma}$ to the frame of rays $\gamma_{1} \cup \gamma_{2}$ and conjugates diffeomorphism $\left.f\right|_{V_{\omega}}$ with the diffeomorphism $\phi_{m, k, n}$, e.g. [5, Statement 10.35 , p. 243]. In this case, we may identify the conjugated objects everywhere below.

Notice that $\gamma_{1}^{0} \times\{0\}$ corresponds to one of unstable separatrices, say $\ell_{1}^{u}$, of the saddle point. Let $\gamma_{2}^{j} \times\{\rho\}$ be another separatrix $\ell_{2}^{u}$, where 
$j \in\{0, \ldots, k-1\}$ and $\rho \in\{0, \ldots, m-1\}$. Then

$$
(\rho, m)=1
$$

due to connectivity of the ambient surface $S_{g}$. Moreover, as $h_{\omega}$ conjugates $f$ with $\phi_{m, k, n}$, it follows from (3.1) that for every $\tau \in\{0, \ldots, k-1\}$

$$
\gamma_{1}^{\tau} \times\{w\} \quad \text { and } \quad \gamma_{2}^{(\tau+j) \bmod k} \times\{w+\rho \bmod m\}
$$

are stable and unstable manifolds of the same saddle point, so the parameters $j$ and $\rho$ determine the are responsible for division of separatrices into stable-unstable submanifolds of the same saddle.

Notice that the choice of $j$ and $\rho$ depends on the order which $h_{\omega}$ maps $\gamma_{1}^{0} \times\{0\}$ and $\gamma_{2}^{j} \times\{\rho\}$ to separatrices of the saddle point. It exchange those separatrices, so $\gamma_{1}^{0} \times\{0\}$ will correspond to $\ell_{2}^{u}$, and $\gamma_{2}^{j^{\prime}} \times\left\{\rho^{\prime}\right\}$ to $\ell_{1}^{u}$, then the pairs $(j, \rho)$ and $\left(j^{\prime}, \rho^{\prime}\right)$ are related by the formulas:

$$
j+j^{\prime}+1=k, \quad \rho+\rho^{\prime}+1=m .
$$

This leads to the formulas

$$
\left(k, j^{\prime}+1\right)=(k, j), \quad\left(m, \rho^{\prime}+1\right)=(m, \rho),
$$

guaranteeing that in (1.3) the periods $m_{\alpha_{1}}, m_{\alpha_{2}}$ do not depend on the order of separatrices.

By Theorem 1.1, the non-wandering set of $f$ contains exactly two source orbits $\mathcal{O}_{\alpha_{1}}, \mathcal{O}_{\alpha_{2}}$ such that $\operatorname{cl}\left(\ell_{1}^{u}\right)=\ell_{1}^{u} \cup \alpha_{1}$, and $\operatorname{cl}\left(\ell_{2}^{u}\right)=\ell_{2}^{u} \cup \alpha_{2}$. Thus

$$
W_{\mathcal{O}_{\omega}}^{s}=S_{g} \backslash \operatorname{cl}\left(W_{\mathcal{O}_{\sigma}}^{s}\right) \text {. }
$$

If we remove from our surface $S_{g}$ the closures of $m_{\sigma}$ stable manifolds, then we get $m$ disks (the basins of the sinks). Since each stable manifold locally separates two such discs on the supporting surface, it follows that every stable manifold will included twice to the boundaries of the disks after cutting. Thus, the boundary of each disk consists of $\frac{2 m_{\sigma}}{m}=2 k$ stable manifolds so that disk can be regarded as $2 k$-gon (see Figures 3.1 and 3.2 on the left).

The stable separatrices are called $s_{1}$ - and $s_{2}$-curves, the unstable separatrices (they are located on the rays of the frames $\gamma_{1}$ and $\gamma_{2}$ ) are called u-curves frames, and the segments connecting the vertices of the polygon with its center are called t-curves. Thus, this (colored) curves divide every polygon into the triangles with $s_{i^{-}}, t-, u$-sides. Let us enumerate these triangles as it shown on Figures 3.1 and 3.2 on the left.

As $u$-sides belonging to the rays

$$
\gamma_{1}^{\tau} \times\{w\}, \quad \text { and } \quad \gamma_{2}^{(\tau+j) \bmod k} \times\{(w+\rho) \bmod m\}
$$



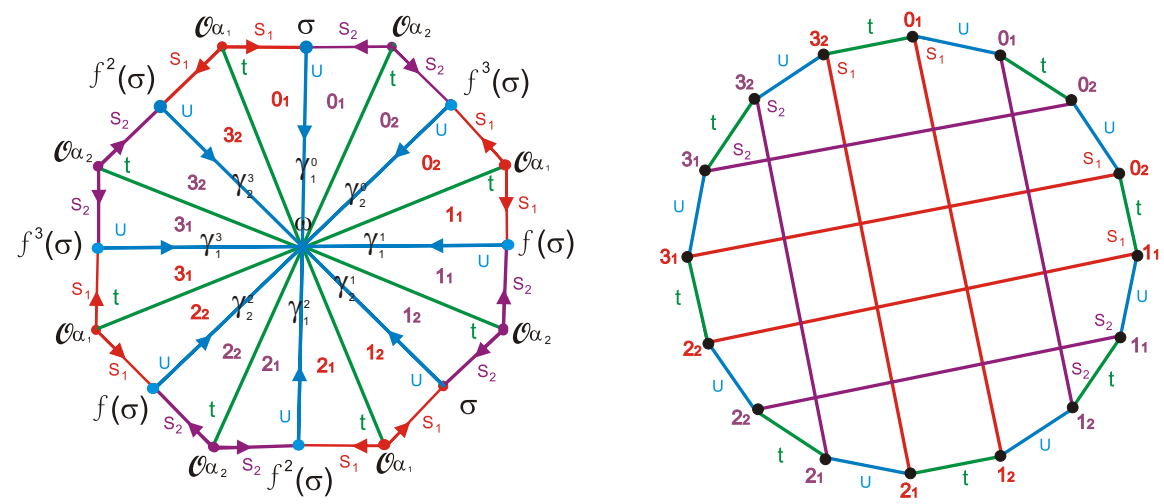

FigurE 3.1. The octagon $\Pi$ that is the closure of the sink basin of the diffeomorphism $f \in G$ (on the left) and the four-color graph $T_{f}$ constructed on it (on the right). Here $m=1, k=4, n=1, j=1, \rho=0$

are separatrices of the same saddle point of $f$, it follows that in order to get the surface $S_{g}$ from the polygons $\Pi_{0}, \ldots, \Pi_{m-1}$ we have to identify the pairs of those sides of polygons which are transversal to this pair of the separatrices.

To compute periods of source points we associate a four-color graph with the diffeomorphism $f$ in the following way (see for details, for example, [7] and $[4])$ :

1) the vertices of the graph $T_{f}$ one-to-one correspond to the triangular regions;

2) two vertices of the graph are incident to the edge of color $s_{1}, s_{2}, t$ or $u$ if the triangular areas corresponding to these vertices have a common $s_{1}, s_{2}, t$ or $u$ side (see Figure 3.1 and 3.2 on the right).

Denote by $B_{f}$ the set of vertices of the graph $T_{f}$ and by $\Delta_{f}$ the set of triangles in the partition of the polygon. Let also $\pi_{f}: \Delta_{f} \rightarrow B_{f}$ be a one-to-one correspondence between the set of triangular domains of the diffeomorphism $f$ and the set of vertices of the graph $T_{f}$. Then $f$ induces an automorphism $P_{f}=\pi_{f} \circ f \circ \pi_{f}^{-1}$ of the set of vertices and edges of the graph $T_{f}$. Moreover,

- the set of sink points of the diffeomorphism $f$ is in a one-to-one correspondence with the set of $t u$-cycles of the graph $T_{f}$;

- the set of saddle points of the diffeomorphism $f$ is in a one-to-one correspondence with the set of $s u$-cycles of the graph $T_{f}$; 
- the set of source points of the diffeomorphism $f$ is in a one-to-one correspondence with the set of $t s$-cycles of the graph $T_{f}$.

Thus, to determine the period $m_{\alpha_{i}}$ of the point $\alpha_{i}, i=1,2$, we have to calculate the number of $s_{i}$ t-cycles. As every such cycle is an image of an other such cycle by $f$, we see that all cycles must have the same period. Hence, the length of each such cycle is some even number (as edges $s_{i}$ and $t$ follow one after other), which we denote by $2 \lambda_{i}$. Notice that the number of $s_{i^{-}}$and $t$-edges in all $s_{i} t$-cycles equals $2 \mathrm{~km}$ then $m_{\alpha_{i}}$ is calculated by the formula

$$
m_{\alpha_{i}}=\frac{k m}{\lambda_{i}}
$$
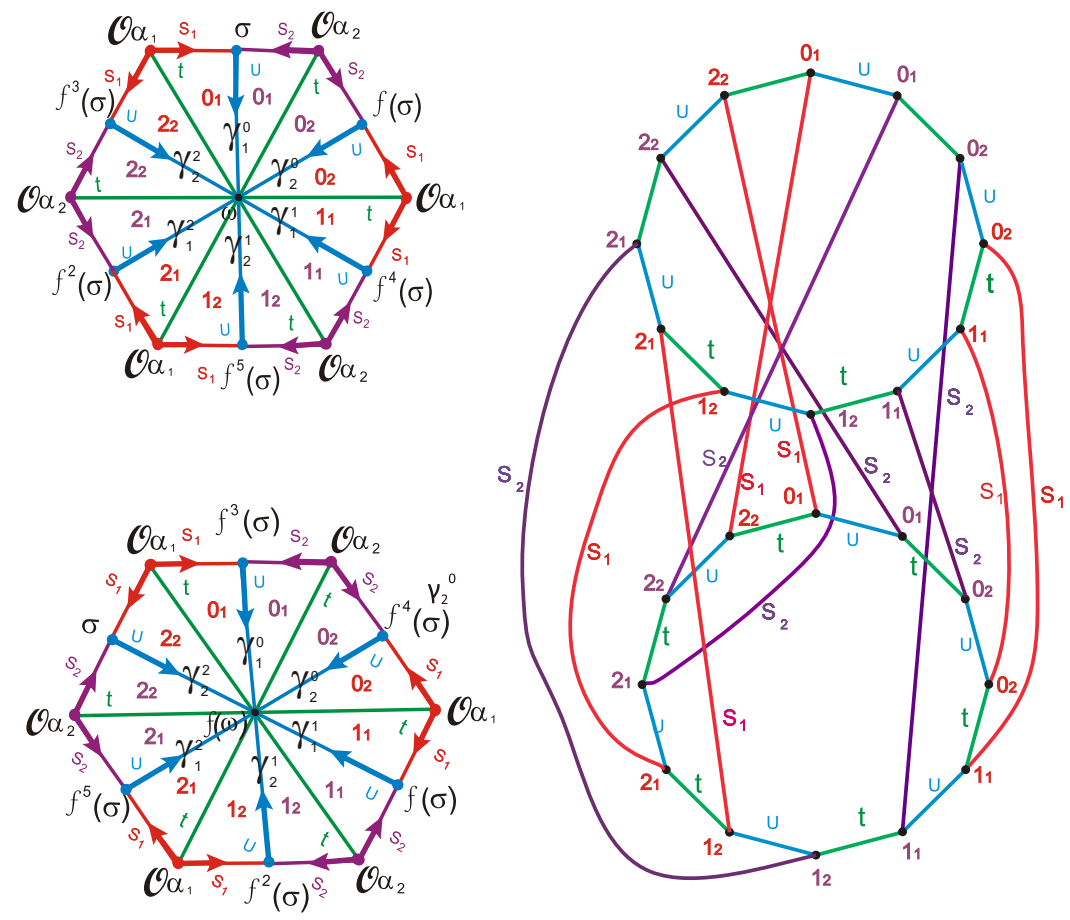

Figure 3.2. Hexagons $\Pi$ and $f(\Pi)$ which are the closures of the sinks basins of the diffeomorphism $f \in G$ (left) and the four-color graph $T_{f}$ (right) constructed from them. Here $m=2, k=3, n=1, j=2$, and $\rho=1$ 
Now we will calculate the length of $s_{1} t$-cycle starting from the $s_{1}$-edge $\left(0_{1}, j_{2}\right)$. We get the following sequence of the vertices

$$
\begin{aligned}
0_{1} & \rightarrow j_{2} \rightarrow((j+1) \bmod k)_{1} \rightarrow((2 j+1) \bmod k)_{2} \rightarrow \\
& \rightarrow(2(j+1) \bmod k)_{1} \rightarrow \cdots \rightarrow\left(\lambda_{1}(j+1) \bmod k\right)_{1},
\end{aligned}
$$

where $(\tau)_{i}$ correspond to the separatrice $\gamma_{i}^{\tau}, i=1,2$. Since that sequence constitute a cycle, we obtain that

$$
\lambda_{1}(j+1) \bmod k=0, \quad \quad \lambda_{1} \rho \bmod m=0,
$$

whence

$$
\lambda_{1}(j+1)=l k, \quad \lambda_{1} \rho=r m
$$

for some $l, r$.

Let $A=(k, j+1)$. Then $k=p A, j+1=q A$, where $(p, q)=1$. Hence,

$$
\lambda_{1}=\frac{l p}{q}=\frac{r m}{\rho} .
$$

As $\lambda_{1}$ is a natural, $(p, q)=1$, and $(\rho, m)=1$, it follows that $l=\mu q$ and $r=\nu \rho$. Hence $\lambda_{1}=\mu p=\nu m$ and $(\mu, \nu)=1$, since $\lambda_{1}$ is the minimal number with the property $\lambda_{1}=\tilde{\mu} p=\tilde{\nu} m$ for some natural $\tilde{\mu}, \tilde{\nu}$.

Let $B=(p, m)$ then $p=x B, m=y B$, where $(x, y)=1$. Therefore $\mu x=\nu y, \mu=y, \nu=x$, and $\lambda_{1}=y p$. Thus

$$
m_{\alpha_{1}}=\frac{k m}{\lambda_{1}}=\frac{k m}{y p}=\frac{p A m}{y p}=A B=(k, j+1)\left(\frac{k}{(k, j+1)}, m\right) .
$$

A similar construction for $\alpha_{2}$ gives $m_{\alpha_{2}}=(k, j)\left(\frac{k}{(k, j)}, m\right)$. By (1.2),

$$
m+(k, j+1)\left(\frac{k}{(k, j+1)}, m\right)+(k, j)\left(\frac{k}{(k, j)}, m\right)-k m=2-2 g .
$$

In an addition, every collection of natural numbers $m \in \mathbb{N}, k \in \mathbb{N}$, $j \in\{0, \ldots, k-1\}$ can be realized by an admissible four-color graph, which in turn, due to [7], allows to construct a diffeomorphism $f \in G\left(S_{g}\right)$ with a periodic data of the form (1.3) on a surface of genus

$$
g=1+\frac{1}{2}\left((k-1) m-(k, j+1)\left(\frac{k}{(k, j+1)}, m\right)-(k, j)\left(\frac{k}{(k, j)}, m\right)\right) .
$$

\section{REFERENCES}

[1] A.N. Bezdenezhnyh, V.Z. Grines. Realization of gradient-like diffeomorphisms of twodimensional manifolds. Sel. Math. Sov., translation from Differential and integral equations (Russian), 11(1):19-23, 1985.

[2] Yu. G. Borisovich, N. M. Bliznyakov, Y. A. Izrailevich, T. N. Fomenko. Introduction to the topology, volume 296. Moscow, Higher school, 1980. 
[3] J. Franks. Homology and dynamical systems. CBSM Regional Conf. Ser. in Math. 49, Amer. Math. Soc., Providence, R.I., 1982.

[4] V. Grines, D. Malyshev, O. Pochinka, S. Zinina. Efficient algorithms for the recognition of topologically conjugate gradient-like diffeomorhisms. Regular and Chaotic Dynamics, 21(2):189-203, 2016.

[5] V. Grines, T. Medvedev, O. Pochinka. Dynamical Systems on 2- and 3-Manifolds., volume 364. Springer International Publishing Switzerland., 2016.

[6] V. Grines, O. Pochinka, S. Van Strien. Realization of gradient-like diffeomorphisms of twodimensional manifolds. arXiv, 2017.

[7] V. Z. Grines, S. Kh. Kapkaeva, O. V. Pochinka. A three-colour graph as a complete topological invariant for gradient-like diffeomorphisms of surfaces. Sbornik Mathematics, 205(10):19-46, 2014.

[8] A Li, J Yorke. Period three implies chaos. Amer. Math. Monthly, (82):985-992, 1975.

[9] E. Nozdrinova, O. Pochinka. On periodic data of polar 2-diffeomorphisms with one saddle orbit. Differential equations and their applications in mathematical modeling: materials of the XIII International Scientific Conference (Saransk, July 12-16, 2017), 408-417, 2017.

[10] J. Palis, W. di Melo. Geometric theory of dynamical systems. An introduction. Berlin, Heidelberg, Springer-Verlag, 1982.

[11] Brown R.F. The Lefschetz fixed point theorem, volume 186. Scott, Foresman and Company, Glenview,IL, 1971.

[12] A.N. Sharkovsky. Coexistence of cycles of a continuous map on itself. Ukrainian mathematical journal, 16(1):61-71, 1964.

[13] S. Smale. Differentiable dynamical systems. Bull. Amer. Math. Soc., 73(6):747-817, 1967.

Received: September 11, 2017, accepted: June 26, 2018.

Elena Nozdrinova

National Research University "Higher School of Economics"

Email: maati@mail.ru

ORCID: orcid.org/0000-0001-5209-377X

Olga Pochinka

National Research University "Higher School of Economics"

Email: olga-pochinka@yandex.ru

ORCID: orcid.org/0000-0002-6587-5305 